\title{
Growth, Structure and Pattern Formation for Thin Films
}

\author{
Russel E. Caflisch
}

Received: 11 January 2007 / Revised: 20 April 2008 / Accepted: 28 April 2008 /

Published online: 13 June 2008

(C) Springer Science+Business Media, LLC 2008

\begin{abstract}
An epitaxial thin film consists of layers of atoms whose lattice properties are determined by those of the underlying substrate. This paper reviews mathematical modeling, analysis and simulation of growth, structure and pattern formation for epitaxial systems, using an island dynamics/level set method for growth and a lattice statics model for strain. Epitaxial growth involves physics on both atomistic and continuum length scales. For example, diffusion of adatoms can be coarse-grained, but nucleation of new islands and breakup for existing islands are best described atomistically. In heteroepitaxial growth, mismatch between the lattice spacing of the substrate and the film will introduce a strain into the film, which can significantly influence the material structure, for example leading to formation of quantum dots. Technological applications of epitaxial structures, such as quantum dot arrays, require a degree of geometric uniformity that has been difficult to achieve. Modeling and simulation may contribute insights that will help to overcome this problem. We present simulations that combine growth and strain showing the structure of nanocrystals and the formation of patterns in epitaxial systems.
\end{abstract}

Keywords Epitaxial growth · Level set method · Island dynamics · Lattice statics · Strain energy $\cdot$ Nanocrystals $\cdot$ Quantum dots

\section{Simulation of Epitaxial Growth}

Epitaxy is the growth of a thin film on a substrate in which the crystal properties of the film are inherited from those of the substrate. Since an epitaxial film can (at least in principle) grow as a single crystal without grain boundaries or other defects, this method produces crystals of the highest quality. In spite of its ideal properties, epitaxial growth is still challenging to mathematically model and numerically simulate because of the wide range of

We acknowledge support from the Focus Center Research Program (FCRP)—Center on Functional Engineered Nano Architectonics (FENA).

R.E. Caflisch $(\bowtie)$

Department of Mathematics, UCLA, Los Angeles, USA

e-mail:vxavxa@gmail.com 
length and time scales that it encompasses, from the atomistic scale of Angstroms and picoseconds to the continuum scale of microns and seconds. This papers reviews our work on simulation of epitaxial growth and of strain in epitaxial system, with applications to the structure of nanocrystals and the formation of patterns on epitaxial surfaces. For simulation of growth we use an island dynamics model with a level set simulation method. Atomistic strain is computed from a linearized lattice statics model.

\subsection{Epitaxial Growth}

The geometry of an epitaxial surface consists of step edges and island boundaries, across which the height of the surface increases by one crystal layer, and adatoms which are weakly bound to the surface. Epitaxial growth involves deposition, diffusion and attachment of adatoms on the surface. Deposition is from an external source, such as a molecular beam. The principal dimensionless parameter (for growth at low temperature) is the ratio $D /\left(a^{4} F\right)$, in which $a$ is the lattice constant and $D$ and $F$ are the adatom diffusion coefficient and deposition flux. It is conventional to refer to this parameter as $D / F$, with the understanding that the lattice constant serves as the unit of length. Typical values for $D / F$ are in the range of $10^{4}$ to $10^{8}$.

The models that are typically used to describe epitaxial growth include the following: Molecular dynamics (MD) consists of Newton's equations for the motion of atoms on an energy landscape. A typical Kinetic Monte Carlo (KMC) method simulates the dynamics of the epitaxial surface through the hopping of adatoms along the surface. The hopping rate comes from an Arrhenius rate of the form $e^{-E / k T}$ in which $E$ is the energy barrier for going from the initial to the final position of the hopping atom. Island dynamics models, one of the subjects of this article, describe the surface through continuum scaling in the lateral directions but atomistic discreteness in the growth direction. Continuum equations approximate the surface using a smooth height function $h=h(x, y, t)$, obtained by coarse graining in all directions. Rate equations describe the surface through a set of bulk variables without spatial dependence.

The island dynamics model described here is solved using a level set simulation method. Within the level set approach [28, 29, 40], the union of all boundaries of islands of height $k+1$, can be represented by the level set $\varphi=k$, for each $k$. For example, the boundaries of islands in the submonolayer regime then correspond to the set of curves $\varphi=0$. Growth of these islands is described by a smooth evolution of the function $\varphi$.

Validation of the island dynamics/level set method will be detailed in this article by comparison to results from an atomistic KMC model. The KMC model employed is a simple cubic pair-bond solid-on-solid (SOS) model [46]. In this model, atoms are randomly deposited at a deposition rate $F$. Any surface atom is allowed to move to its nearest neighbor site at a rate $r$ that is determined by $r=r_{0} \exp \left\{-\left(E_{S}+n E_{N}\right) / k_{B} T\right\}$, where $r_{0}$ is a prefactor which is chosen to be $10^{13} \mathrm{~s}^{-1}, k_{B}$ is the Boltzmann constant, and $T$ is the surface temperature. $E_{S}$ and $E_{N}$ represent the surface and nearest neighbor bond energies, and $n$ is the number of nearest neighbors.

Level set methods have been used for a number of thin film growth problems that are related to the applications described below. In [13] a level set method was used to simulate coarsening, and in [43] a level set method was used to describe spiral growth in epitaxy. A general level set approach to material processing problems, including etching, deposition and lithography, was developed in [1,2] and [3]. A similar method was used in [30] for deposition in trenches and vias. 


\subsection{Island Dynamics}

Burton, Cabrera and Frank [7] developed the first detailed theoretical description for epitaxial growth. In this "BCF" model, the adatom density solves a diffusion equation with an equilibrium boundary condition $\left(\rho=\rho_{\mathrm{eq}}\right)$, and step edges (or island boundaries) move at a velocity determined from the diffusive flux to the boundary. Modifications of this theory were made, for example in $[6,11,16,20,23]$, to include line tension, edge diffusion and nonequilibrium effects. These are "island dynamics" models, since they describe an epitaxial surface by the location and evolution of the island boundaries and step edges. They employ a mixture of coarse graining and atomistic discreteness, since island boundaries are represented as smooth curves that signify an atomistic change in crystal height.

Adatom diffusion on the epitaxial surface is described by a diffusion equation of the form

$$
\partial_{t} \rho-D \nabla^{2} \rho=F-2(d / d t) N_{\text {nuc }}
$$

in which the last term represents loss of adatoms due to nucleation and desorption from the epitaxial surface has been neglected. Attachment of adatoms to the step edges and the resulting motion of the step edges are described by boundary conditions at an island boundary (or step edge) $\Gamma$ for the diffusion equation and a formula for the step-edge velocity $v$.

For the boundary conditions and velocity, several different models are used. The simplest of these is

$$
\begin{aligned}
& \rho=\rho_{*}, \\
& v=D[\partial \rho / \partial n]
\end{aligned}
$$

in which the brackets indicate the difference between the value on the upper side of the boundary and the lower side. Two choices for $\rho_{*}$ are $\rho_{*}=0$, which corresponds to irreversible aggregation in which all adatoms that hit the boundary stick to it irreversibly, and $\rho_{*}=\rho_{\mathrm{eq}}$ for reversible aggregation. For the latter case, $\rho_{\mathrm{eq}}$ is the adatom density for which there is local equilibrium between the step and the terrace [7].

Line tension and edge diffusion can be included in the boundary conditions and interface velocity as in

$$
\begin{aligned}
\partial \rho / \partial n_{ \pm} & =D_{T}\left(\rho_{ \pm}-\rho_{*}\right)-\mu \kappa, \\
v & =D_{T} \mathbf{n} \cdot[\nabla \rho]+\beta \rho_{* s s}+\left(\mu / D_{E}\right) \kappa_{s s},
\end{aligned}
$$

in which $\rho_{ \pm}$and $\partial \rho / \partial n_{ \pm}$are the limiting values of the adatom density and its normal derivative at a step from the upper $(+)$ and lower $(-)$ terraces, $\mathbf{n}$ is the normal direction at a step (pointing into the lower terrace), $\kappa$ is curvature, $s$ is the variable along the boundary, and $D_{E}$ is the coefficient for diffusion along and detachment from the boundary. The term $\beta \rho_{*_{s s}}$ was derived in [20] due to edge diffusion.

A snapshot of the results from a typical level-set simulation is shown in Fig. 1. This figure shows the epitaxial surface, consisting of islands of various heights, after deposition of 40 layers. Numerical details on implementation of the level set method for thin film growth are provided in [12].

\subsection{Nucleation and Submonolayer Growth}

For the case of irreversible aggregation, a dimer (consisting of two atoms) is the smallest stable island, and the nucleation rate is

$$
\frac{d N_{\mathrm{nuc}}}{d t}=D \sigma_{1}\left\langle\rho^{2}\right\rangle,
$$


Fig. 1 Snapshot of a typical level-set simulation after deposition of 40 layers

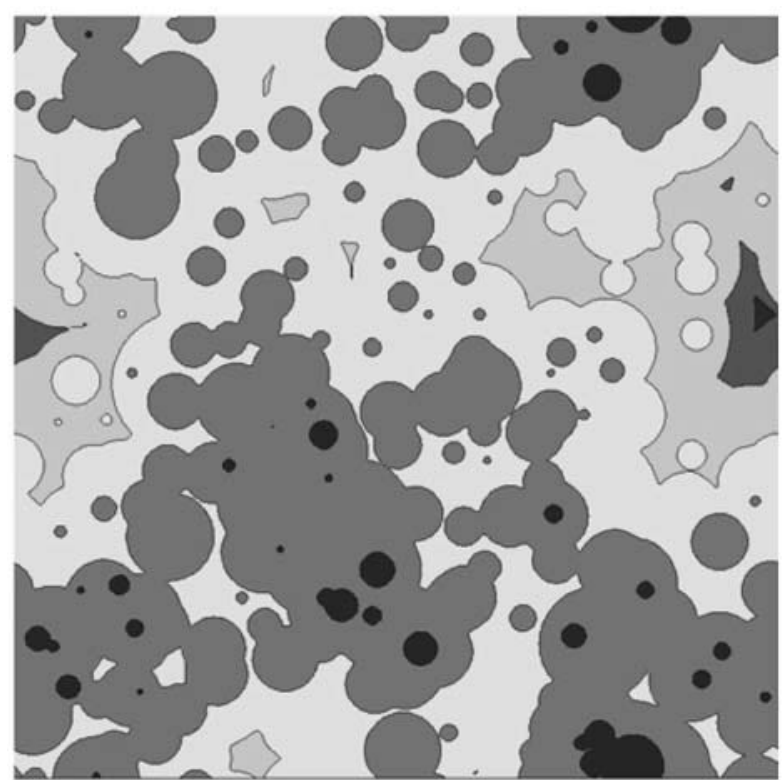

where $\langle\cdot\rangle$ denotes the spatial average of $\rho(\mathbf{x}, t)^{2}$ and

$$
\sigma_{1}=\frac{4 \pi}{\ln [(1 / \alpha)\langle\rho\rangle D / F]}
$$

is the adatom capture number as derived in [5, 45]. Without the factor $\sigma_{1},(4)$ describes the rate of collisions for a system of uniformly distributed particles that do not stick together; the factor $\sigma_{1}$ provides the leading order correction for particles that stick together. The parameter $\alpha$ reflects the island shape, and $\alpha \simeq 1$ for compact islands. Expression (4) for the nucleation rate implies that the time of a nucleation event is chosen deterministically. Whenever $N_{\text {nuc }} L^{2}$ passes the next integer value ( $L$ is the system size), a new island is nucleated. Numerically, this is realized by raising the level-set function to the next level at a number of grid points chosen to represent a dimer.

The choice of the location of the new island is determined by probabilistic choice with spatial density proportional to the nucleation rate $\rho^{2}$. This probabilistic choice constitutes an atomistic fluctuation that must be retained in the level set model for faithful simulation of the epitaxial morphology. For growth with compact islands, computational tests have shown additional atomistic fluctuations can be omitted [36].

Additions to the basic level set method, such as terms that represent finite lattice constant effects and edge diffusion (not the term $\kappa_{s s}$ but a surrogate term that has a similar effect), are easily included [37]. The level set method with these corrections is in excellent agreement with the results of KMC simulations. For example, Fig. 2 shows the island size distribution (ISD)

$$
n_{s}=\frac{\Theta}{s_{a v}^{2}} g\left(s / s_{a v}\right),
$$

where $n_{s}$ is the scaled density of islands of size $s, s_{a v}$ is the average island size, and $g(x)$ is a scaling function. The top panel of Fig. 2 is for irreversible attachment; the other two panels 
Fig. 2 The island size distribution, as given by KMC (squares) and LS (circles) methods, in comparison with STM experiments (triangles) on $\mathrm{Fe} / \mathrm{Fe}(001)$ [44]. The reversibility increases from top to bottom

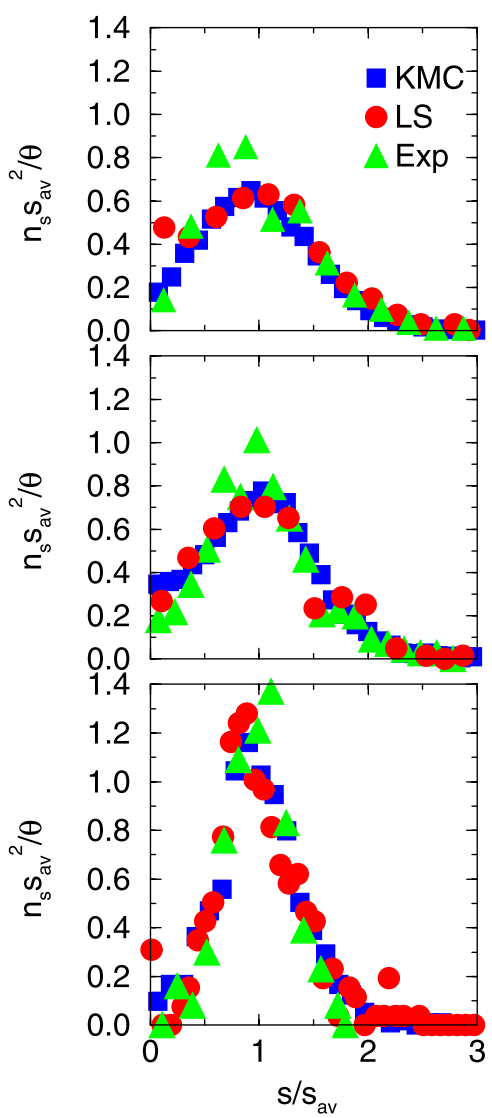

include reversibility that will be discussed below. All three panels show excellent agreement between the results from level set simulations, KMC and experiment.

\subsection{Multilayer Growth}

In ideal layer-by-layer growth, a layer is completed before nucleation of a new layer starts. In this case, growth on subsequent layers would essentially be identical to growth on previous layers. In reality, however, nucleation on higher layers starts before the previous layer has been completed and the surface starts to roughen. This roughening transition depends on the growth conditions (i.e., temperature and deposition flux) and the material system (i.e., the value of the microscopic parameters). At the same time, the average lateral feature size increases in higher layers, which we will refer to as coarsening of the surface.

These features of multilayer growth and the effectiveness of the level set method in reproducing them is illustrated in Fig. 3 which shows the island number density $N$ as a function of time for two different values of $D / F$ from both a level set simulation and from KMC. The results show near perfect agreement. The KMC results were obtained with a value for the edge diffusion that is $1 / 100$ of the terrace diffusion constants. The island density decreases as the film height increases which implies that the film coarsens.

The simulation results presented above have been for the case of irreversible aggregation. If aggregation is reversible, the KMC method must simulate a large number of detachment 
Fig. 3 Island densities $N$ on each layer for $D / F=10^{6}$ (lower panel) and $D / F=10^{7}$ (upper panel) obtained with the level-set method and KMC simulations. For each data set there are 10 curves in the plot, corresponding to the 10 layers

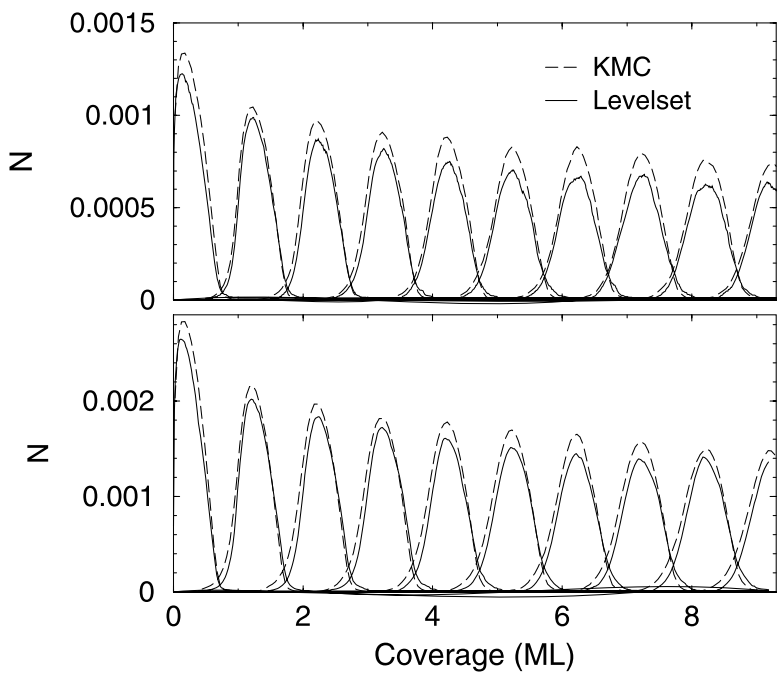

and reattachment events that can slow down the simulations significantly. On the other hand, in a level set simulation these events can be directly replaced by their time average and therefore the simulation only needs to include detachment events that do not lead to a subsequent reattachment, making the level set method much faster than KMC, as shown in [33]. Reversibility can be included in the level set method using the boundary conditions (2) with $\rho_{*}=\rho_{\mathrm{eq}}$ in which $\rho_{\mathrm{eq}}$ depends on the local environment of the island, in particular the edge atom density [8]. For islands consisting of only a few atoms, however, the stochastic nature of detachment becomes relevant and is included through random detachment and breakup for small islands, as detailed in [33].

\section{Strain in Thin Films}

\subsection{Numerical Simulations for Thin Films}

In heteroepitaxial growth, a thin film of one material (e.g., Ge) is grown on top of a substrate of a second material (e.g., $\mathrm{Si}$ ), with perfect, single crystalline structure in both materials and with the lattice structure of the film determined by the substrate. If the lattice constants $a_{f}$ and $a_{s}$ for the film and substrate are different (e.g., $a_{\mathrm{Ge}}=1.04 \times a_{\mathrm{Si}}$ ) then strain is generated in the film. Figure 4 illustrates the horizontal compression, as well as the resulting vertical expansion, in the lattice of the film atoms, when they are placed on a substrate of smaller lattice constant. This strain has important effects on the material structure, as well as on its electronic properties.

For this system, it is most convenient to define the atomic displacement relative to a single reference lattice, for example the equilibrium lattice of the substrate, so that the displacement $\mathbf{u}$ in the film is defined relative to a nonequilibrium reference lattice. The bond displacement $\mathbf{d}^{\mathbf{k} \pm}$ is then

$$
\mathbf{d}^{\mathbf{k} \pm}(\mathbf{i})=\left(d_{1}^{k \pm}, d_{2}^{k \pm}, d_{3}^{k \pm}\right)=D_{k}^{ \pm} \mathbf{u}(\mathbf{i})-\epsilon \mathbf{e}_{\mathbf{k}} \chi
$$


Fig. 4 (a) Equilibrium lattice for film with lattice size $a_{f}$.

(b) Equilibrium lattice for substrate with lattice size $a_{s}$. (c) The reference (non-equilibrium) lattice in which strain is introduced due to lattice mismatch
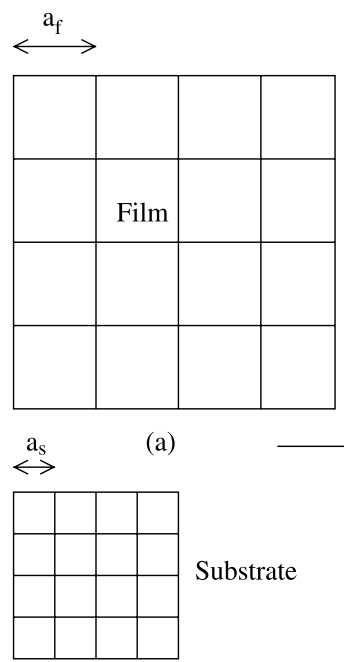

(a)

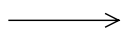

Substrate

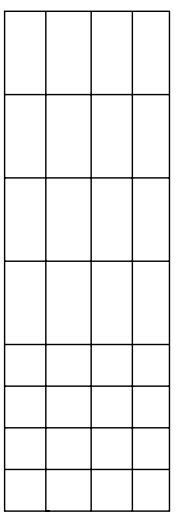

(c)

(b)

and the discrete strain components at a point $\mathbf{i}$ are defined as

$$
S_{k \ell}^{p q}=\frac{D_{k}^{p} u_{\ell}+D_{\ell}^{q} u_{k}-\epsilon \delta_{k \ell} \chi}{2} .
$$

In these equations $D_{k}^{+}$and $D_{k}^{-}$denote forward and backward difference operators, $\epsilon=$ $\frac{a_{f}-a_{s}}{a_{s}}$ is the relative lattice displacement and $\chi$ is 0 in the substrate and 1 in the film. The resulting discrete strain equations have a force of size $\epsilon$ along the film/substrate interface. The atomistic strain energy at a point $\mathbf{i}$ has the form

$$
E(\mathbf{i})=\alpha \sum_{p= \pm, k=1,2}\left(S_{k k}^{p}\right)^{2}+\sum_{p= \pm, q= \pm}\left(2 \beta\left(S_{12}^{p q}\right)^{2}+\gamma S_{11}^{p} S_{22}^{q}\right)
$$

where the elastic constant $\alpha, \beta$ and $\gamma$ are chosen so that the model is consistent with continuum elasticity, namely,

$$
\alpha=C_{11} / 4, \quad \beta=C_{44} / 4, \quad \gamma=C_{12} / 4
$$

in which $C_{i j}$ are the Voigt constants.

The total energy can be obtained by summing up all the energy densities to get

$$
\mathcal{E}^{\text {total }}=\sum_{\mathbf{i}} E(\mathbf{i})
$$

Minimization of this energy leads to a force balance equation

$$
\frac{\partial \mathcal{E}^{\text {total }}}{\partial u_{k}}=0, \quad \text { for } k=1,2 .
$$

Away from boundaries and interfaces, these equations are equivalent to a finite difference approximation to the equations of continuum elasticity. At a step on a boundary or interface, 
there are deviations from the continuum boundary conditions which can be interpreted as force distributions on the boundary. Details of the energy density and the discrete force balance equations can be found in [14, 39].

Computational solution of the strain equations can be computationally challenging. We apply two methods to reduce the computational complexity. First we use an artificial boundary condition along a plane in the substrate that is everywhere below the film. This greatly reduces the extent of the computational domain, with no loss of accuracy [19]. Second, we apply an algebraic multigrid method to solve the strain equations, that greatly accelerates the computations [9]. Similar methods have been developed and implemented by Smereka and Russo [38]. Simulations of island dynamics models including elastic effects have been performed by Hauser, Jabbour and Voigt [18], as well as by Niu et al. [27].

\section{Modeling and Simulation for the Structure of Nanocrystals}

Layered nanocrystals consist of a core of one material surrounded by a shell of a second material. Synthesis of layered nanocrystals with precise control over their size and shape has been achieved by a number of research groups [10, 22, 25, 32] and provides an effective method for designing material systems with desired optoelectronic properties [22].

Because of the small size of these systems, their atomic structure is epitaxial in many cases. Lattice mismatch between the materials in the core and shell leads to elastic strain in a layered nanocrystal. This strain has both structural and optoelectronic consequences. If the strain is large enough, then it is relieved by irregular growth of the shell [22]; i.e., the epitaxial structure is lost. As a result, the shell may break off from the core [22].

The present study from [4] employs a simple model for the structure and strain of a layered nanocrystal. Simulation of this model for a range of geometric and elastic parameters shows that there is a critical shell size at which strain has maximal influence.

\subsection{Core/Shell Model}

Denote the lattice constants in the core and shell as $l_{c}$ and $l_{s}$, respectively. For bonds connecting a core atom and a shell atom, the rest length is taken to be the average $\left(l_{c}+l_{s}\right) / 2$. Similarly the elastic coefficients for the bonds connecting a core atom and a shell atom are taken to be the averages of the elastic coefficients for the pure materials.

The significant geometric parameters are the core radius $r_{c}$, the shell thickness $r_{s}$ and the lattice mismatch

$$
\epsilon=\frac{l_{c}-l_{s}}{l_{c}}
$$

The core consists of atoms whose lattice position $x$ (before displacement) satisfies $|x| \leq r_{c}$, and the shell consists of atoms with $r_{c}<|x| \leq r_{c}+r_{s}$, as shown in Fig. 5.

\subsection{Critical Thickness: Simulation Results}

Computational results are presented here from minimization of the total elastic energy (after removing degenerate modes corresponding to translation and rotation), corresponding to balance of all of the forces in the system, for 2D (circular, or equivalently rods of infinite length) and 3D (spherical) nanocrystals. For the harmonic potentials used here, this amounts to solving a linear system of equations, in which the forcing terms come from the lattice 
mismatch $\epsilon$. The simulation results include values of the displacements, the forces and the energy density. Graphical results will be presented for the last of these. As a figure of merit for the atomistic strain field in a nanocrystal, we shall use the maximum value $E_{m}$ of the discrete energy density. Since the energy at each atom consists of elastic energy and bond energy, the maximum elastic energy may be a good indicator of strain-driven instability.

\subsubsection{Elastic Energy Density}

Figure 6 show the elastic energy density of 3D layered nanocrystals, of fixed core size $r_{c}$ for various values of shell thickness $r_{s}$. In these simulations, the shell has thickness values $r_{s}=$ 1,2 and 7 monolayers, on a core of radius $r_{c}=8$ monolayers. For all of these simulations, the elastic constants are $\alpha=5, \beta=1$ and $\gamma=3$ and lattice mismatch is $\epsilon=0.04$.

In this figure, the gray scale ranges from black for $E=0$ to white for $E=E_{m}$ in which $E_{m}$ is the largest value of $E$ among the three subfigures; i.e., the scales are same for the different subfigures. The black region outside of each nanocrystal is a vacuum where there is no energy. Figure 6 shows that the energy is concentrated in the region of the shell, along the interface with the core. As the shell thickness increases, the strain energy becomes more concentrated near the shell/core interface, even though the maximum energy density decreases for larger shell thickness. In addition the largest values of the energy density are close to the diagonal.

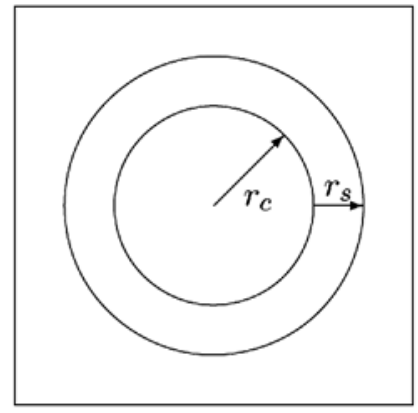

(a) $2 \mathrm{D}$

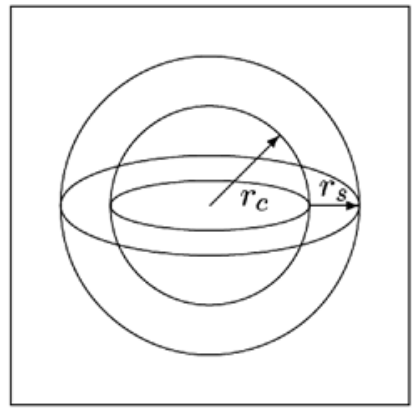

(b) $3 \mathrm{D}$

Fig. 5 Basic geometry of core/shell nanocrystal model

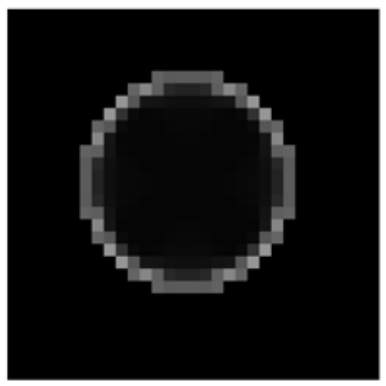

(a)

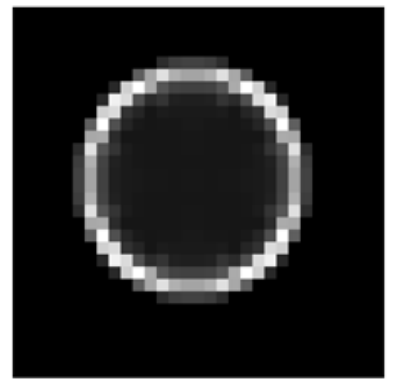

(b)

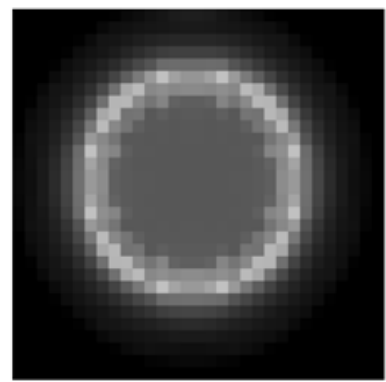

$\mathrm{c}$

Fig. 6 Elastic energy density on an equatorial cross section for 3D layered nanocrystals with core size $r_{c}=8$ monolayers and with shell thickness $r_{s}$ of size (a) 1 monolayer, (b) 2 monolayers and (c) 7 monolayers 


\subsubsection{Critical Thickness}

Figure 7 shows the maximum energy density for a layered nanocrystal, as a function of shell thickness $r_{s}$, for fixed values of the other parameters, core size $r_{c}$ and elastic constants $\alpha, \beta$, $\gamma$ and $\epsilon$. Figure 7 shows that the maximum energy density increases with increasing shell thickness $r_{s}$ up to a critical shell thickness $r_{s}^{*}$. For $r_{s}>r_{s}^{*}$, the maximum energy density is decreasing as a function of $r_{s}$. The general similarity between the critical shell thickness in $2 \mathrm{D}$ and $3 \mathrm{D}$ is indicative of the robustness of this result. The physical core radius of $\mathrm{CdSe} / \mathrm{CdS}$ core/shell nanocrystal is ranging from $11.5 \AA$ to $19.5 \AA$ which is equivalent to core radius of 3 monolayers to 6 monolayers, since one full monolayer is approximately $3.5 \AA$ [32].

Our simulations show weak sensitivity of critical shell thickness $r_{s}^{*}$ on the core radius $r_{c}$. The critical thickness $r_{s}^{*}$ is uniformly 2 monolayers as long as the core size is big enough. In simulation, for smaller core size than 3 monolayers for $2 \mathrm{D}$ layered nanocrystals and 5

Fig. 7 Maximum energy density $E_{m}$ vs. shell thickness $r_{s}$ for (a) 2D and (b) 3D nanocrystal of core radius $r_{c}=8$ monolayers

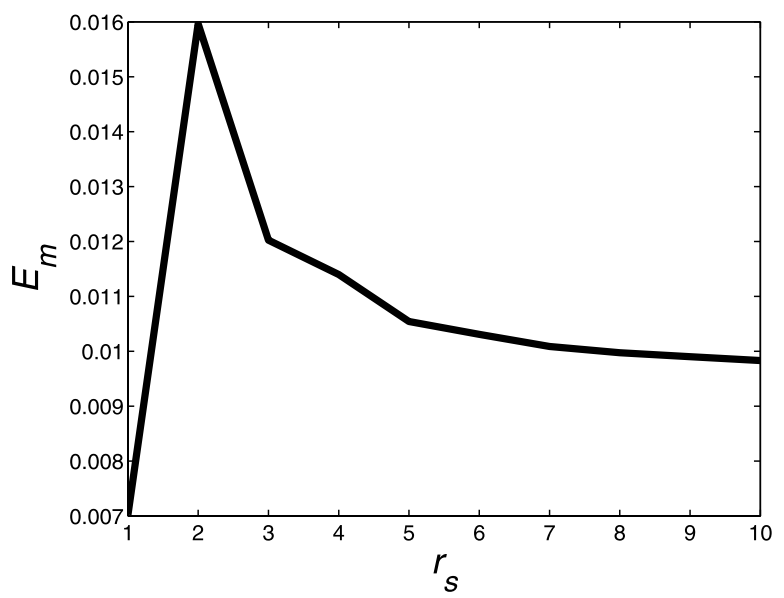

(a)

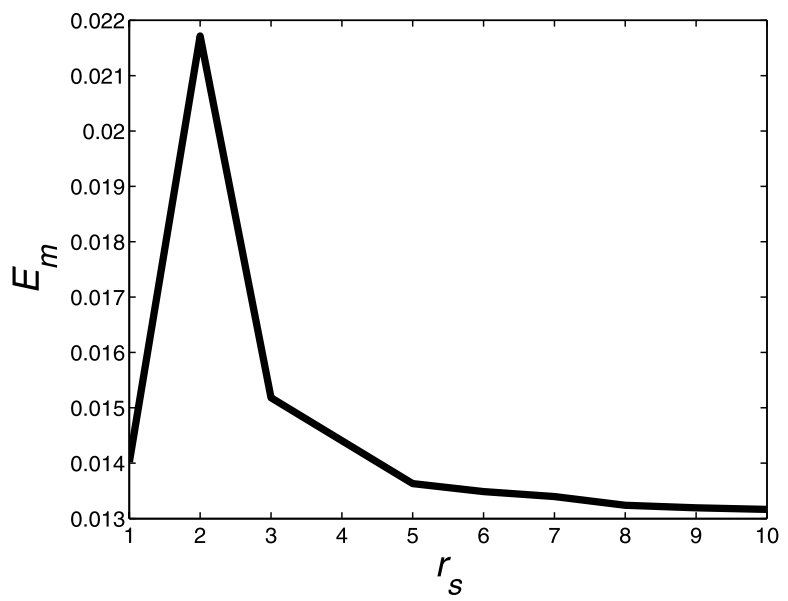

(b) 
monolayers for 3D layered nanocrystals, the maximum elastic energy density $E_{m}$ occurs at 1 monolayer of shell thickness $r_{s}$. We also find that the critical shell thickness $r_{s}^{*}$ is roughly independent of the lattice misfit $\epsilon$ and the elastic parameters $\alpha, \beta$ and $\gamma$.

\section{Patterns in Epitaxial Systems}

\subsection{Self-Assembly}

Highly ordered and uniformly sized nano patterns play an increasingly important role for many technological applications. A critical factor for the performance of all such devices is that the patterns are all within a certain size range (which depends on the material), and that the dots are essentially all equal in size. It is therefore the focus of a large number of studies to understand the formation and growth of nano patterns (for recent reviews, see [41, 42]), and to control their formation and size distribution.

There are various approaches to obtaining arrays of equally sized and spaced nano patterns. In the top-down approach, islands nucleate in previously fabricated nucleation sites. But structures as small as a few nm are difficult to obtain with standard lithographic techniques. In the bottom-up approach, kinetic and/or thermodynamic factors spontaneously lead to the formation of quantum dots $[15,17,24]$. Guided or directed self-assembly is somewhere in-between. In this approach, the goal is to control pattern formation by manipulating the epitaxial growth process. For example, introduction of subsurface dislocation arrays introduces a long-range strain field, which alters the potential energy surface (PES), changing both the adsorption energy $E_{\text {ad }}$ and the transition energy $E_{\text {trans }}$ of the PES [31,35]. The results presented here from [26] simulate the process of directed self-assembly, starting from a spatially varying PES.

\subsection{Epitaxial Growth with Spatially Varying Potential Energy Surface}

In this section, we discuss simulations that demonstrate that a properly modified PES for adatom diffusion can lead to self organization of nano patterns. Adatom diffusion is described by a rate for surface diffusion, which is $D=D_{0} \exp \left(-\Delta E / k_{B} T\right)$, where $D_{0}$ is a prefactor (chosen to be $10^{13} \mathrm{~s}^{-1}$ ), $k_{B}$ is the Boltzmann constant, $T$ is the temperature, and $\Delta E$ is the energy barrier for surface diffusion, given by $\Delta E=E_{\text {trans }}-E_{\text {ad }}$. We study growth systematically as a function of $E_{\text {ad }}$ and $E_{\text {trans }}$, which are treated as independent parameters.

For a surface with a spatially varying, anisotropic PES, the diffusion equation (1) becomes

$$
\frac{\partial \rho}{\partial t}=F+\nabla \cdot(\mathbf{D} \nabla \rho)-2 \frac{d N}{d t}+\nabla \cdot\left(\frac{\rho}{k_{B} T} \mathbf{D}\left(\nabla E_{\mathrm{ad}}\right)\right) .
$$

In (14), $\mathbf{D}$ is a diffusion tensor where the diagonal entries are labeled $D_{i}(\mathbf{x})$ and $D_{j}(\mathbf{x})$, and correspond to diffusion along the two directions $i$ and $j$. For simplicity no other direction for diffusion is included (but could easily be incorporated). $F$ is the deposition flux, $d N / d t$ is the nucleation rate, and the last term is the thermodynamic drift, where $k_{B}$ is the Boltzmann constant, and $T$ is the temperature. On island boundaries there is rapid attachment and detachment of adatoms, so that the correct boundary condition is $\rho(\mathbf{x})=\rho_{\mathrm{eq}}\left(D_{\mathrm{det}}(\mathbf{x}), \mathbf{x}\right)$, where $D_{\text {det }}(\mathbf{x})$ is a (spatially varying) detachment rate [8]. The nucleation rate is given by [37]

$$
d N / d t=\sigma_{1}\left\langle\left[\left(D_{i}(\mathbf{x})+D_{j}(\mathbf{x})\right) / 2\right] \rho^{2}(\mathbf{x})\right\rangle,
$$

where $\sigma_{1}$ is the capture number as in (5), and the average $\langle\cdot\rangle$ is taken over all lattice sites. 


\subsection{Simulation Results}

We assume a simple sinusoidal variation of $E_{\text {ad }}$ and $E_{\text {trans }}$. More precisely, for the results shown in Fig. 8, we assume that the diffusion constant varies between $D=10^{5} \mathrm{~s}^{-1}$ and $D=10^{7} \mathrm{~s}^{-1}$ along the $i$-direction, and that in fact $\operatorname{LOG}_{10} D$ varies sinusoidally. Diffusion is isotropic but spatially varying, and we use the notation $D=D_{i}(\mathbf{x})=D_{j}(\mathbf{x})$. A schematic of the variations of the PES is shown in the bottom panels of Fig. 8. The periodicity of the variation of the PES in the $i$-direction was chosen to be 50 atomic spacings. We also use a simplified spatial variation of $D_{\text {det }}$, and vary it between $422 \mathrm{~s}^{-1}$ and $750 \mathrm{~s}^{-1}$. Smaller or larger numbers for $D_{\text {det }}$, or even a constant $D_{\text {det }}$ in the same range, lead to very similar results.

The results shown in Fig. 8 correspond to the thermodynamic limit (left panel), where only $E_{\text {ad }}$ is varied, and the kinetic limit (right panel), where only $E_{\text {trans }}$ is varied. For the particular choices presented here, the spatial variation of the diffusion constant $D$ is identical in both cases. The PES is varied only along the $i$-direction, and is constant along the $j$-direction. It is immediately evident from the morphologies that islands almost exclusively form along stripes in either limit. But in the kinetic limit, the islands are rather large, while they are much smaller in the thermodynamic limit. Closer inspection shows that the positions of most islands are inverted. In fact the islands nucleate in the region of fast diffusion (low potential energy barrier) in the kinetic limit, but nucleate in the region of slow diffusion in the thermodynamic limit, and that correspondingly all the mass is in these regions.

The explanation for this is the following: In the nucleation rate $d N / d t$ in (14), the parameter $\sigma_{1}$ is essentially constant, so that $d N / d t$ increases either when $D$ increases, or when $\rho(\mathbf{x})$ increases. In the kinetic limit (without a thermodynamic drift), $\rho(\mathbf{x})$ is spatially constant (at least before islands start acting as sinks on the surface, which is the case in the nucleation phase), so that the nucleation rate is dominant in regions where $D$ is large. However, once a thermodynamic drift is present, the adatom concentration is not constant, and is in fact largest in regions where $E_{\text {ad }}$ has its minimum. If the drift term is large enough, $d N / d t$ is dominated by a large $\rho$, which is in regions where $D$ is small (large barrier).

We can now also understand why the islands are much larger in the kinetic limit: Here, nucleation is determined by a large diffusion constant. But the diffusion constant $D$ also determines a characteristic length $l_{\text {char }} \sim D^{\chi}$, which characterizes the size of and spacing between islands. The positive exponent $\chi$ depends on the degree of reversibility (i.e., $D_{\text {det }}$ and $F$ ) [34]. This means that in regions of large $D$, islands are on average larger and fewer.

Fig. 8 Morphologies as obtained in the kinetic limit (right) and the thermodynamic limit (left) (top panels). A schematic of the envelope of the underlying variations of the PES is shown at the bottom for each case. Note that each period of the sinusoidal variation corresponds to 50 lattice constants

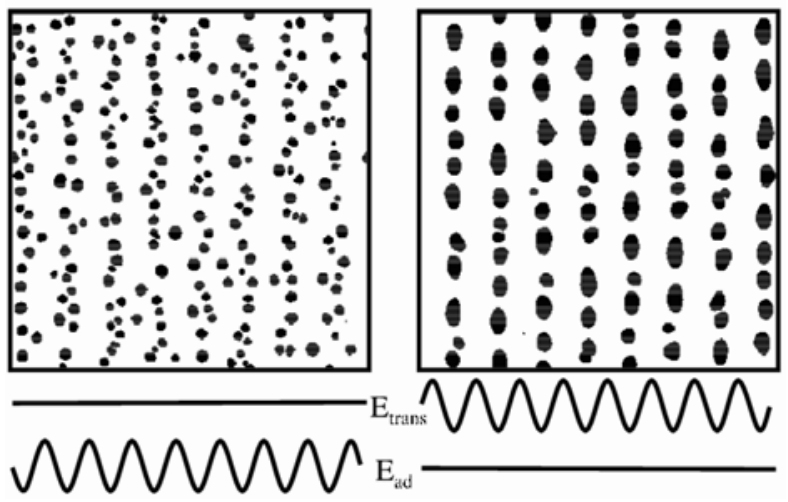


Fig. 9 Morphologies at coverages $\Theta=0.1 \mathrm{ML}$ (left) and $\Theta=0.3 \mathrm{ML}($ right $)$ obtained with a PES (below) that has a much narrower variation

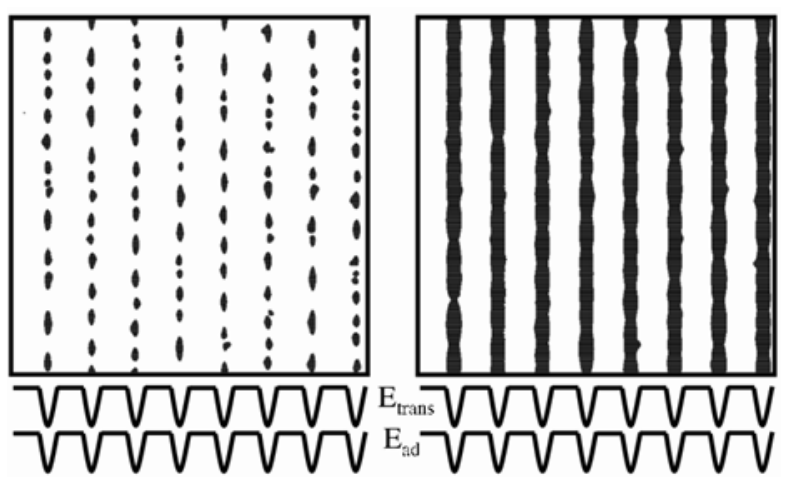

On the other hand, in the thermodynamic limit, islands nucleate in the region of small $D$, where $l_{\text {char }}$ is smaller, and hence there are more and smaller islands.

The morphologies shown so far were all obtained at a sub-monolayer pre-coalescence coverage of $\Theta=0.2 \mathrm{ML}$ and with a PES that varies sinusoidally. In Fig. 9 we show the morphology at different coverages obtained with PES that varies more sharply in certain regions, and is essentially constant in others. At $\Theta=0.1 \mathrm{ML}$, the islands are aligned even better than in the previously discussed cases. Moreover, at $\Theta=0.3 \mathrm{ML}$, all the islands that are aligned along the $j$-direction have coalesced in this direction, while they do not touch at all along the $i$-direction. In fact, we get a very regular array of one-dimensional, monolayer-high nano-wires on the surface. Our simulations suggest a new mechanism by which quantum wires can be obtained, with a width that can be much smaller.

\section{Conclusions}

The simulations described above have established the validity of the level set method for simulation of epitaxial growth. Moreover, the level set method makes possible simulations that would be difficult for atomistic methods such as KMC; e.g., systems with large rates of attachment/detachment due to strain [33]. This method can now be used with confidence in many applications that include epitaxy along with additional phenomena and physics.

Atomistic strain due to lattice mismatch in heteroepitaxy is an important feature of thin films. The mathematical model and computational method described here make possible effective simulation of epitaxial systems with strain even in three dimensions; e.g., [27]. This may allow application to many epitaxial phenomena of scientific and technological interest. Two examples presented here are the structure of nanocrystals and pattern formation on an epitaxial surface.

We have examined the elastic energy density of a nanocrystal and the corresponding critical shell thickness. The simulation results presented above are for a highly idealized model of a layered nanocrystal. The robustness of these results with respect to variation of dimension, geometry and material parameters suggests that these results are qualitative and generally applicable.

The results on pattern formation suggest an approach to guiding self-assembly of nano patterns. Application of this approach, even in simulation, will require several additional ingredients, including microscopic models of elasticity and of the strain dependence of the PES and other properties. Also, strain induced changes of the PES due to the developing surface morphologies should be included in a more comprehensive model [21]. 


\section{References}

1. Adalsteinsson, D., Sethian, J.A.: A level set approach to a unified model for etching, deposition, and lithography. 1. Algorithms and two-dimensional simulations. J. Comput. Phys. 120, 128-144 (1995)

2. Adalsteinsson, D., Sethian, J.A.: A level set approach to a unified model for etching, deposition, and lithography. 2. 3-Dimensional simulations. J. Comput. Phys. 122, 348-366 (1995)

3. Adalsteinsson, D., Sethian, J.A.: A level set approach to a unified model for etching, deposition, and lithography. 3. Redeposition, reemission, surface diffusion, and complex simulations. J. Comput. Phys. 138, 193-223 (1997)

4. Bae, Y., Caflisch, R.E.: Strain in layered nanocrystals. Eur. J. Appl. Math. 18, 571-585 (2007)

5. Bales, G.S., Chrzan, D.C.: Dynamics of irreversible island growth during submonolayer epitaxy. Phys. Rev. B 50, 6057-6067 (1994)

6. Bales, G.S., Zangwill, A.: Morphological instability of a terrace edge during step flow growth. Phys. Rev. B 41, 5500-5508 (1990)

7. Burton, W.K., Cabrera, N., Frank, F.C.: The growth of crystals and the equilibrium structure of their surfaces. Philos. Trans. R. Soc. Lond. Ser. A 243, 299-358 (1951)

8. Caflisch, R.E., E, W., Gyure, M., Merriman, B., Ratsch, C.: Kinetic model for a step edge in epitaxial growth. Phys. Rev. E 59, 6879-6887 (1999)

9. Caflisch, R.E., Lee, Y.-J., Shu, S., Xiao, Y., Xu, J.: An application of multigrid methods for a discrete elastic model for epitaxial systems. J. Comput. Phys. 219, 697-714 (2006)

10. Cao, Y.-W., Banin, U.: Synthesis and characterization of InAs/InP and InAs/CdSe core/shell nanocrystals. Angew. Chem. Int. Ed. 38, 3692-3694 (1999)

11. Cermelli, P., Jabbour, M.E.: Possible mechanism for the onset of step-bunching instabilities during the epitaxy of single-species crystalline films. Phys. Rev. B 75, 165409 (2007)

12. Chen, S., Kang, M., Merriman, B., Caflisch, R.E., Ratsch, C., Fedkiw, R., Gyure, M.F., Osher, S.J.: Level set method for thin film epitaxial growth. J. Comput. Phys. 167, 475-500 (2001)

13. Chopp, D.L.: A level-set method for simulating island coarsening. J. Comput. Phys. 162, 104-122 (2000)

14. Connell, C., Caflisch, R.E., Luo, E., Simms, G.D.: The elastic field of a surface step: The MarchenkoParshin formula in the linear case. J. Comput. Appl. Math. 196, 368-386 (2006)

15. Eaglesham, D.J., Cerullo, M.: Dislocation-free Stranski-Krastanov growth of Ge on Si(100). Phys. Rev. Lett. 64, 1943-1946 (1990)

16. Ghez, R., Iyer, S.S.: The kinetics of fast steps on crystal surfaces and its application to the molecular beam epitaxy of silicon. IBM J. Res. Develop. 32, 804-818 (1988)

17. Guha, S., Madhukar, A., Rajkumar, K.C.: Onset of incoherency and defect introduction in the initial stages of molecular beam epitaxial growth of highly strained $\operatorname{In}_{x} \mathrm{Ga}_{1-x}$ As on $\mathrm{GaAs}(100)$. Appl. Phys. Lett. 57, 2110-2112 (1990)

18. Hauser, F., Jabbour, M.E., Voigt, A.: A step-flow model for the heteroepitaxial growth of strained, substitutional, binary alloy films with phase segregation: I. Theory. Multiscale Model. Simul. 6, 158-189 (2007)

19. Lee, S., Caflisch, R.E., Lee, Y.-J.: Artificial boundary conditions for continuum and discrete elasticity. SIAM J. Appl. Math. 66, 1749-1775 (2006)

20. Li, B., Caflisch, R.E.: Analysis of island dynamics in epitaxial growth. Multiscale Model. Simul. 1, 150-171 (2002)

21. Lung, M.T., Lam, C.-H., Sanders, L.M.: Island, pit, and groove formation in strained heteroepitaxy. Phys. Rev. Lett. 95, 086102 (2005)

22. Manna, L., Schoer, E.C., Li, L.-S., Alivisatos, A.P.: Epitaxial growth and photochemical annealing of graded CdS/ZnS shells on colloidal CdSe nanorods. J. Am. Chem. Soc. 124, 7136-7145 (2002)

23. Michely, T., Krug, J.: Islands, Mounds and Atoms. Springer, Berlin (2004)

24. Mo, Y.-W., Savage, D.E., Swartzentruber, B.S., Lagally, M.G.: Kinetic pathway in Stranski-Krastanov growth of Ge on Si(001). Phys. Rev. Lett. 65, 1020-1023 (1990)

25. Mokari, T., Banin, U.: Synthesis and properties of CdSe/ZnS core/shell nanorods. Chemistry of Materials 15(20), 3955-3960 (2003)

26. Niu, X., Vardavas, R., Caflisch, R.E., Ratsch, C.: Level set simulation of directed self-assembly during epitaxial growth. Phys. Rev. B, Brief Report 74 (2006). Art. No. 193403

27. Niu, X., Lee, Y.J., Caflisch, R.E., Ratsch, C.: Optimal capping layer thickness for stacked quantum dots. Preprint (2008)

28. Osher, S., Sethian, J.A.: Front propagation with curvature dependent speed: Algorithms based on Hamilton-Jacobi formulations. J. Comput. Phys. 79, 12-49 (1988)

29. Osher, S.J., Fedkiw, R.P.: Level Set Methods and Dynamic Implicit Surfaces. Springer, New York (2002) 
30. O’Sullivan, P.L., Baumann, F.H., Gilmer, G.H., Torre, J.D., Shin, C.S., Petrov, I., Lee, T.Y.: Continuum model of thin film deposition incorporating finite atomic length scales. J. Appl. Phys. 92, 3487-3494 (2002)

31. Penev, E., Kratzer, P., Scheffler, M.: Effect of strain on surface diffusion in semiconductor heteroepitaxy. Phys. Rev. B 64, 085401 (2001)

32. Peng, X., Schlamp, M.C., Kadavanich, A.V., Alivisatos, A.P.: Epitaxial growth of highly luminescent $\mathrm{CdSe} / \mathrm{CdS}$ core/shell nanocrystals with photostability and electronic accessibility. J. Am. Chem. Soc. 119, 7019-7029 (1997)

33. Petersen, M., Ratsch, C., Caflisch, R.E., Zangwill, A.: Level set approach to reversible epitaxial growth. Phys. Rev. E 64(061602), U231-U236 (2001)

34. Ratsch, C., Šmilauer, P., Zangwill, A., Vvedensky, D.D.: Submonolayer epitaxy without a critical nucleus. Surf. Sci. 329, L599-L604 (1995)

35. Ratsch, C., Seitsonen, A.P., Scheffler, M.: Strain dependence of surface diffusion: Ag on $\operatorname{Ag}(111)$ and Pt(111). Phys. Rev. B 55, 6750-6753 (1997)

36. Ratsch, C., Gyure, M.F., Chen, S., Kang, M., Vvedensky, D.D.: Fluctuations and scaling in aggregation phenomena. Phys. Rev. B 61, 10598-10601 (2000)

37. Ratsch, C., Gyure, M.F., Caflisch, R.E., Gibou, F., Petersen, M., Kang, M., Garcia, J., Vvedensky, D.D.: Level-set method for island dynamics in epitaxial growth. Phys. Rev. B 65(195403), U697-U709 (2002)

38. Russo, G., Smereka, P.: Computation of strained epitaxial growth in three dimensions by kinetic Monte Carlo. J. Comput. Phys. 214, 809-828 (2006)

39. Schindler, A.C., Gyure, M.F., Simms, G.D., Vvedensky, D.D., Caflisch, R.E., Connell, C., Luo, E.: Theory of strain relaxation in heteroepitaxial systems. Phys. Rev. B 67, 075316 (2003)

40. Sethian, J.A.: Level Set Methods and Fast Marching Methods: Evolving Interfaces in Computational Geometry, Fluid Mechanics, Computer Vision, and Materials Science. Cambridge University Press, Cambridge (1999)

41. Shchukin, V.A., Bimberg, D.: Spontaneous ordering of nanostructure on crystal surfaces. Rev. Mod. Phys. 71, 1125-1171 (1999)

42. Stangl, J., Holy, V., Bauer, G.: Structural properties of self-organized semiconductor nanostructures. Rev. Mod. Phys. 76, 725-783 (2004)

43. Smereka, P.: Spiral crystal growth. Physica D 138, 282-301 (2000)

44. Stroscio, J.A., Pierce, D.T.: Scaling of diffusion-mediated island growth in iron-on-iron homoepitaxy. Phys. Rev. B 49, 8522-8525 (1994)

45. Venables, J.: Rate equation approaches to thin film nucleation kinetics. Philos. Mag. 27, 697-738 (1973)

46. Vvedensky, D.D.: Atomistic modeling of epitaxial growth: comparisons between lattice models and experiment. Comput. Mater. Sci. 6, 182-187 (1996) 